\title{
Effect of Tyrosol and Farnesol on Virulence and Antibiotic Resistance of Clinical Isolates of Pseudomonas aeruginosa
}

\author{
Shaymaa Hassan Abdel-Rhman, ${ }^{1}$ Areej Mostafa El-Mahdy, ${ }^{1,2}$ and Mohammed El-Mowafy \\ ${ }^{1}$ Department of Microbiology and Immunology, Faculty of Pharmacy, Mansoura University, Mansoura 35516, Egypt \\ ${ }^{2}$ Department of Pharmaceutical Sciences, Faculty of Pharmacy, Princess Norah Bint Abdulrahman University, \\ Riyadh 11671, Saudi Arabia \\ Correspondence should be addressed to Mohammed El-Mowafy; mohammedasaad7@gmail.com
}

Received 11 September 2015; Revised 31 October 2015; Accepted 14 December 2015

Academic Editor: Stanley Brul

Copyright (c) 2015 Shaymaa Hassan Abdel-Rhman et al. This is an open access article distributed under the Creative Commons Attribution License, which permits unrestricted use, distribution, and reproduction in any medium, provided the original work is properly cited.

\begin{abstract}
Mixed-species biofilms could create a protected environment that allows for survival to external antimicrobials and allows different bacterial-fungal interactions. Pseudomonas aeruginosa-Candida albicans coexistence is an example for such mixed-species community. Numerous reports demonstrated how P. aeruginosa or its metabolites could influence the growth, morphogenesis, and virulence of $C$. albicans. In this study, we investigated how the C. albicans quorum sensing compounds, tyrosol and farnesol, might affect Egyptian clinical isolates of $P$. aeruginosa regarding growth, antibiotic sensitivity, and virulence. We could demonstrate that tyrosol possesses an antibacterial activity against P. aeruginosa ( $10 \mu \mathrm{M}$ inhibited more than $50 \%$ of growth after $16 \mathrm{~h}$ cultivation). Moreover, we could show for the first time that tyrosol strongly inhibits the production of the virulence factors hemolysin and protease in P. aeruginosa, whereas farnesol inhibits, to lower extent, hemolysin production in this bacterial pathogen. Cumulatively, tyrosol is expected to strongly affect $P$. aeruginosa in mixed microbial biofilm.
\end{abstract}

\section{Introduction}

Quorum sensing (QS) compounds are signaling molecules that are released by the cells in a manner depending on their population density. In bacteria, the concentration of these compounds is elevated as the number of bacteria increases. Such autostimulatory compounds induce the population to cooperate in diverse behaviors such as bioluminescence, antibiotic production, virulence, biofilm formation, and sporulation [1]. In Gram-negative bacteria, these compounds are usually acyl homoserine lactones, whereas in Grampositive bacteria, they are often modified peptides [2]. In Candida albicans, farnesol (sesquiterpene derivative) [3] and tyrosol (tyrosine derivative) [4] were identified as the main QS compounds in this pathogenic yeast.

Farnesol is a metabolic product of mevalonate/sterol synthetic pathway in eukaryotes [3]. Weber et al., 2008, have shown that farnesol produced in situ by planktonic C. albicans cultures inhibits biofilm formation [5]. The accumulation of farnesol was found to block the morphological shift from yeast to hyphae form at high cell densities [3], suggesting that Candida pathogenesis may be reduced by farnesol [6]. Contrary to farnesol, tyrosol was found to accelerate the morphological transition from yeasts to hyphae [7].

Quorum sensing signals were found not only to modulate single species bacterial and fungal communications, but also to mediate crosstalk between bacteria and the fungus $C$. albicans [8]. Mixed bacterial-C. albicans coinfections can be associated with an increase in the severity of the disease. For example, coinoculation of C. albicans and Staphylococcus aureus in mice was associated with high mortality in comparison with sole inoculation of $S$. aureus $[9,10]$. The bacterialC. albicans interactions were not restricted to enhancement of virulence of bacterial species but they were also extended to affect the bacterial resistance to antimicrobial agents. For instance, it was demonstrated that the human pathogen $S$. aureus forms larger biofilms with increased resistance to vancomycin when it is cocultured with C. albicans [11]. Biofilms of oral streptococci and C. albicans are similarly 
more resistant to antibiotics than their single species counterparts $[8,10,12]$.

A variety of populations of bacteria and fungi colonizes humans. Interactions between these microbial populations can be useful or detrimental to the host either in healthy state or in the settings of disease $[8,10]$. A significant proportion of human microbial infections are biofilm associated, wherein the formation of mixed-species biofilms could create a protected environment that allows for survival to external assaults and facilitates different bacterial-fungal interactions [8].

The coexistence of Pseudomonas aeruginosa and C. albicans is an example of a pathogenic community. P. aeruginosa is extremely difficult to treat due to its high intrinsic and adaptive antibiotic resistance, ability to form biofilms in chronic infections, and broad arsenal of virulence factors [13]. Several reports indicate that $P$. aeruginosa and $C$. albicans can coexist in different opportunistic infections [14-18], and a variety of different molecular interactions between these two organisms have been investigated [18-22]. In the last years, both pathogenic organisms have been shown to be involved in device-associated nosocomial infections for almost all types of indwelling devices [23].

Therefore in this study we were interested in investigating the possible effect of C. albicans QS compounds, tyrosol and farnesol, on the antibiotic resistance and some of the virulence factors of Egyptian isolates of P. aeruginosa.

\section{Methods}

2.1. Bacterial Strains and Reagents. The strains of P. aeruginosa used in this study were clinical isolates collected from Mansoura University Hospital and obtained from different sources: burn, wound, urine, and pus. All the clinical isolates were collected after approval from the administrative authorities (Research Ethics Committee) in the Faculty of Pharmacy, Mansoura University, Egypt, on 13/10/2014, with the code number $214-70$. The isolates were stored at $-70^{\circ} \mathrm{C}$ till use. Farnesol (Sigma number 277541) and tyrosol (Sigma number 79058) were stored at $-20^{\circ} \mathrm{C}$ before preparation of $500 \mathrm{mM}$ stock solution in DMSO or water for farnesol and tyrosol, respectively. Farnesol stock solution was prepared immediately prior to each experiment and it was added to the final concentration just at the time of inoculation, where control cultures received an equivalent amount of DMSO [24]. Muller-Hinton ( $\mathrm{MH}$ ) broth, $\mathrm{MH}$ agar, and antibiotic sensitivity disks were obtained from Oxoid.

\subsection{P. aeruginosa Growth Assay upon Farnesol or Tyrosol} Treatment. The growth of representative eight Egyptian isolates of $P$. aeruginosa was tested in the presence of different concentrations of tyrosol and farnesol according to [25] with little modification. Briefly, $2 \times 10^{5}$ cells $/ \mathrm{mL}$ of $P$. aeruginosa were inoculated in $\mathrm{MH}$ broth supplemented with different concentrations of farnesol $(10,50,100$, and $200 \mu \mathrm{M})$ or tyrosol $(1.2,2.5,5,10,20,40$, and $80 \mu \mathrm{M})$, employing 96 -well plates in a final volume of $200 \mu \mathrm{L}$. In case of farnesol containing wells, the final volume of DMSO was $1 \%$ the total volume in each well. Farnesol and tyrosol free controls were supplemented with $1 \%$ DMSO and water, respectively. Cultures were allowed to grow at $37^{\circ} \mathrm{C}$ for 16 hours. The growth of the cells was determined by measuring the optical density (OD) at $600 \mathrm{~nm}$ using the absorbance microplate reader, BioTek ELx800. All experiments were carried out in triplicate.

The effect of farnesol and tyrosol on cell viability was also estimated by colony counting. The cell densities, incubation conditions, and the concentrations of tyrosol and farnesol were followed as previously mentioned in the measurement of $\mathrm{OD}_{600 \mathrm{~nm}}$ in the 96-well plates except that the culture volume was adjusted to $2 \mathrm{~mL}$ shaking at $150 \mathrm{rpm}$. After incubation, the cells were harvested by centrifugation at $4000 \mathrm{rpm}$ for $5 \mathrm{~min}$ and washed with $\mathrm{MH}$ broth before being plated on $\mathrm{MH}$ agar for at least $24 \mathrm{~h}$ at $37^{\circ} \mathrm{C}$ to determine their viability. The survival rate of these cultures, expressed as colony forming units (CFU), was compared to farnesol and tyrosol free cultures.

2.3. Disk Diffusion Assay. The susceptibility of $P$. aeruginosa strains to a number of clinically important antibiotics was investigated in the presence of farnesol or tyrosol. Cultures were grown for overnight at $37^{\circ} \mathrm{C}$ with shaking in $\mathrm{MH}$ broth. Aliquots $(100 \mu \mathrm{L})$ were streaked onto $\mathrm{MH}$ agar plates with farnesol final concentration $(10 \mu \mathrm{M})$ or tyrosol final concentration of $(1.2 \mu \mathrm{M})$ followed by application of the following antibiotic-impregnated disks (Oxoid Ltd.). Nucleic acid inhibitors (ciprofloxacin [5 $\mu \mathrm{g}$ ] and norfloxacin $[10 \mu \mathrm{g}]$ ), cell wall synthesis inhibitors (ceftriaxone [30 $\mathrm{g}]$ and cefepime $[30 \mu \mathrm{g}]$ ), intermediary metabolism inhibitors (trimethoprim and sulfamethoxazole [25 $\mu \mathrm{g}]$ ), and protein synthesis inhibitors (erythromycin [15 $\mu \mathrm{g}$ ] and gentamicin $[10 \mu \mathrm{g}])$ were applied to pseudomonas-seeded agar plates at specified concentration of farnesol or tyrosol. Plates were incubated at $37^{\circ} \mathrm{C}$ for $18 \mathrm{~h}$, and the zones of inhibition surrounding the antibiotic disks were measured and recorded [26].

2.4. Effect of Farnesol and Tyrosol on P. aeruginosa Total Proteases and Hemolysin. The effect of farnesol and tyrosol on the production of total protease and hemolysin enzymes of $P$. aeruginosa isolates was performed using previously prepared culture supernatant.

Total protease activity was measured using a casein substrate as previously described [27].

The hemolysin test was carried out by incubating $0.6 \mathrm{~mL}$ of fresh sheep red blood cells $(2 \%)$ with $0.6 \mathrm{~mL}$ of bacterial supernatant at $37^{\circ} \mathrm{C}$ for $2 \mathrm{~h}$. Hemoglobin release was measured at $\mathrm{OD}_{540 \mathrm{~nm}}$. The test was also performed in absence of the bacterial supernatant (negative control) or in presence of SDS $(0.2 \%)$ instead of the bacterial supernatant (positive control) [28].

2.5. Statistical Analysis. Statistical analysis was performed using Student's $t$-test for paired data. Significance was set at 0.1\% using Microsoft Excel.

\section{Results}

3.1. Farnesol and Tyrosol Inhibit the Growth and Viability of $P$. aeruginosa. In this work we evaluated the influence 


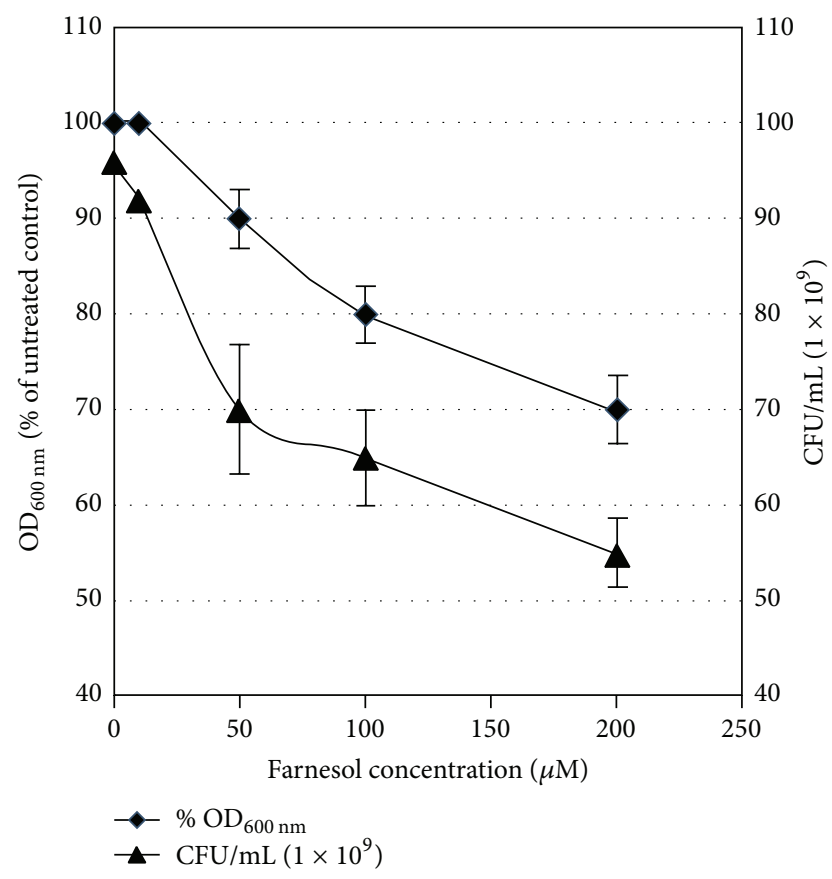

FIgURE 1: Growth-inhibitory effects of farnesol on cells of $P$. aeruginosa (isolate 1). Cells were grown in $\mathrm{MH}$ broth medium containing farnesol at $0,10,50,100$, and $200 \mu \mathrm{M}$. Cell growth was expressed either as \% growth after relating to the untreated cultures (at $\mathrm{OD}_{600 \mathrm{~nm}}$ ) or as number of cells (CFU) counted after $24 \mathrm{~h}$ cultivation on $\mathrm{MH}$ agar. Error bars represent the standard deviation from three independent experiments.

of the quorum sensing compounds of C. albicans (farnesol and tyrosol) on the growth of representative eight Egyptian isolates of $P$. aeruginosa. These results were identical in the eight tested isolates of $P$. aeruginosa. A representative data for the effect of farnesol and tyrosol on one of these isolates (isolate 1) is shown in Figures 1 and 2, respectively. The growth was inhibited after $16 \mathrm{~h}$ by $30 \%$ or more than $50 \%$ in the presence of $200 \mu \mathrm{M}$ farnesol or $10 \mu \mathrm{M}$ tyrosol, respectively. On the other side, $1.2 \mu \mathrm{M}$ tyrosol and $50 \mu \mathrm{M}$ farnesol either had no effect on growth or inhibited it by $10 \%$, respectively.

Both the percent of growth (expressed as $\mathrm{OD}_{600 \mathrm{~nm}}$ ) and the number of viable bacterial cells decreased proportionally with farnesol and tyrosol concentrations, suggesting that both compounds have an antibacterial activity against $P$. aeruginosa.

3.2. Influence of Farnesol and Tyrosol on the Antibiotic Sensitivity of $P$. aeruginosa. Disk diffusion assay showed no detectable difference in the inhibition zones obtained by the discs of tested antibiotics in presence or absence of either of the two investigated QS compounds. These results were further confirmed by data obtained from broth microdilution experiments (data not shown). These experiments were performed using twofold serial dilutions of three of the most widely administrated broad spectrum antibiotics: ceftriaxone, ciprofloxacin, and gentamicin at concentrations of 64000-0.12, 4000-0.004, and 2000-0.002 $\mu \mathrm{g} / \mathrm{mL}$, respectively. In both disk diffusion and broth microdilution experiments,

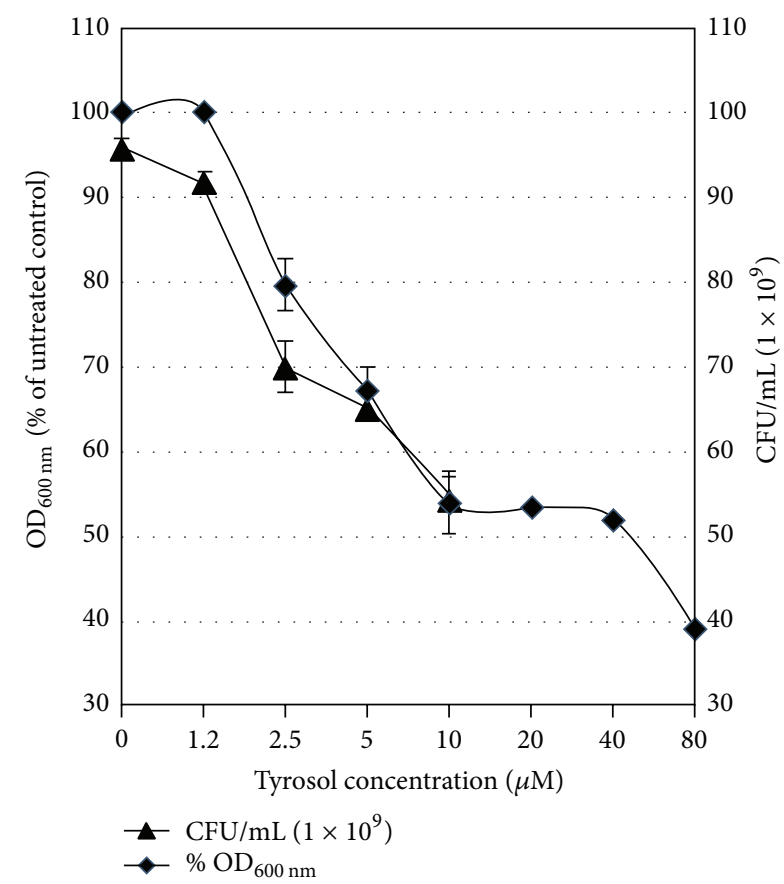

FIgURE 2: Growth-inhibitory effects of tyrosol on cells of $P$. aeruginosa (isolate 1). Cells were grown in $\mathrm{MH}$ broth medium containing tyrosol at $0,1.25,2.5,5,10,20,40$, and $80 \mu \mathrm{M}$. Cell growth was expressed either as \% growth after relating to the untreated cultures (at $\mathrm{OD}_{600 \mathrm{~nm}}$ ) or as number of cells (CFU) counted after $24 \mathrm{~h}$ cultivation on $\mathrm{MH}$ agar. Error bars represent the standard deviation from three independent experiments.

the effect of tyrosol and farnesol was tested at concentrations 1.2 and 10 that affect the growth yield by not more than $10 \%$.

3.3. Effect of Farnesol and Tyrosol on Total Proteases and Hemolysin Production by P. aeruginosa. We next examined the effect of farnesol and tyrosol on the production of total proteases and hemolysin. Treating $P$. aeruginosa isolates with farnesol $(10 \mu \mathrm{M})$ or tyrosol $(1.2 \mu \mathrm{M})$ caused reduction in the production level of hemolysin (Table 1) and total proteases (Table 2).

Tyrosol significantly inhibited the production of hemolysin $(p<0.001)$ and total proteases $(p<0.001)$ in the investigated isolates. Additionally, farnesol significantly inhibited the production hemolysin $(p<0.001)$ without signification inhibition of total proteases.

\section{Discussion}

The interaction between $C$. albicans and $P$. aeruginosa in mixed communities was reported in many studies. From the side of $P$. aeruginosa, the effect of either the bacterial pathogen itself or its metabolites on C. albicans was extensively investigated in numerous studies [11, 18, 29, 30]. Few reports have investigated the effect of coculturing of $C$. albicans or incubation with farnesol on $P$. aeruginosa regarding growth, virulence, and antibiotic resistance of this bacterial pathogen. For example, C. albicans has been shown to enhance the production of $P$. aeruginosa phenazine toxins in cocultured 
TABLE 1: Effect of farnesol and tyrosol on hemolysin production in Egyptian isolates of P. aeruginosa.

\begin{tabular}{|c|c|c|c|c|c|}
\hline \multirow[b]{2}{*}{ Isolate number } & \multirow{2}{*}{$\begin{array}{c}\text { Control } \\
\text { Hemolysis percent } \\
\text { caused by the isolate }\end{array}$} & \multicolumn{2}{|c|}{ Tyrosol effect } & \multicolumn{2}{|c|}{ Farnesol effect } \\
\hline & & $\begin{array}{c}\text { Before } \\
\text { normalization }\end{array}$ & $\begin{array}{c}\text { After } \\
\text { normalization }\end{array}$ & $\begin{array}{c}\text { Before } \\
\text { normalization }\end{array}$ & After normalization \\
\hline 1 & 23.5 & 4.8175 & -79.5 & 6.4625 & -72.5 \\
\hline 2 & 12.3 & 1.1931 & -90.3 & 2.46 & -80 \\
\hline 3 & 27.4 & 22.8242 & -16.7 & 24.4956 & -10.6 \\
\hline 4 & 60.7 & 11.6544 & -80.8 & 33.8099 & -44.3 \\
\hline 5 & 59.8 & 3.887 & -93.5 & 17.94 & -70 \\
\hline 6 & 34.1 & 22.7447 & -33.3 & 30.008 & -12 \\
\hline 7 & 72.3 & 19.521 & -73 & 7.5915 & -89.5 \\
\hline 8 & 38.8 & 30.458 & -21.5 & 29.4104 & -24.2 \\
\hline 9 & 89.9 & 36.4095 & -59.5 & 21.576 & 76 \\
\hline 10 & 100 & 1 & -99 & 25 & -75 \\
\hline 11 & 10 & 9 & -10 & 9 & -10 \\
\hline 12 & 79.4 & 6.352 & -92 & 29.2192 & -63.2 \\
\hline 13 & 71 & 20.519 & -71.1 & 47.357 & -33.3 \\
\hline 14 & 22.3 & 15.2978 & -31.4 & 12.9117 & -42.1 \\
\hline 15 & 50.5 & 13.5845 & -73.1 & 14.7965 & -70.7 \\
\hline 16 & 81.1 & 7.3801 & -90.9 & 20.275 & -75 \\
\hline 17 & 7.6 & 6.802 & -10.5 & 7.448 & -2 \\
\hline 18 & 92.3 & 27.69 & -70 & 50.765 & -45 \\
\hline 19 & 90 & 21.51 & -76.1 & 34.65 & -61.5 \\
\hline 20 & 31.7 & 21.4609 & -32.3 & 10.1123 & -68.1 \\
\hline
\end{tabular}

The values in the control column represent the levels of hemolysin production by the isolates $(\mathrm{SD}=30.6)$, indicated by hemolysis percent, without addition of farnesol and tyrosol. The values in the columns of tyrosol and farnesol stand for either nonnormalized values (SD = 10.2 and 13.5 for tyrosol and farnesol, resp.) or normalized values. The latter values indicate the percent of either decrease (negative values) or increase (positive values) in the production of hemolysin after normalization to untreated samples (control). All values represent the mean of three independent experiments.

colony biofilms [18, 31]. Moreover, the quorum sensingregulated phenazines in $P$. aeruginosa were stimulated when cultured with $C$. albicans [31]. Farnesol was shown to inhibit the swarming motility in $P$. aeruginosa [32].

In this work we were interested to determine how the $C$. albicans QS compounds (tyrosol and farnesol) might affect the antibiotic sensitivity and production of the virulence factors, hemolysin and total proteases in Egyptian isolates of $P$. aeruginosa. To our knowledge, the effect of tyrosol on $P$. aeruginosa is not yet reported. Care was taken to perform firstly growth studies of a concentration-dependent manner for farnesol and tyrosol. Our results showed that the growth of $P$. aeruginosa was inhibited by more than $50 \%$ in the presence of $10 \mu \mathrm{M}$ tyrosol, while $1.2 \mu \mathrm{M}$ was the highest concentration that did not exhibit any effect on the growth of $P$. aeruginosa. On the other side, farnesol inhibited the growth of $P$. aeruginosa by 10 and $30 \%$ at concentrations of 50 and $200 \mu \mathrm{M}$, respectively. Previous report showed that farnesol did not affect the growth of $P$. aeruginosa strain PA14 at a concentration of $250 \mu \mathrm{M}$ [24]. It is important to mention that the corresponding physiological relevant concentration of tyrosol and farnesol produced by stationary-phase $C$. albicans cultures is about 350-400 $\mu \mathrm{M}[4]$ and $50 \mu \mathrm{M}[5,33]$, respectively. This indicates that the C. albicans QS molecule, tyrosol, would certainly affect the growth of $P$. aeruginosa in mixed biofilms. Our results of the inhibitory effects of farnesol are consistent with the observed antibacterial effect of farnesol against other bacterial species such as Staphylococcus aureus [26], Streptococcus mutans [34], and Staphylococcus epidermidis [35].

For further investigation of the effect of tyrosol and farnesol on antibiotic sensitivity and the production of hemolysin and total proteases in the isolates of $P$. aeruginosa, subinhibitory concentrations that were not exceeding the corresponding physiological relevant concentration were further used in this study (1.2 and $10 \mu \mathrm{M}$ for tyrosol and farnesol, resp.).

Jabra-Rizk et al., 2006, have shown that the antibiotic sensitivity in $S$. aureus was increased in the presence of farnesol [26]; they related this synergistic effect to the possible membrane permeabilizing action of farnesol that subsequently increases the sensitivity of $S$. aureus to antibiotics. Indeed, many compounds that are able to increase the sensitivity to antibiotics might have outer membrane permeabilizing action [36]. Our results showed that neither tyrosol nor farnesol, at their subinhibitory concentrations, was found to have any effect on the antibacterial activity of different antibiotics on $P$. aeruginosa. We might also conclude that farnesol and tyrosol do not have membrane permeabilizing action on $P$. aeruginosa, which is in contrast to the membrane 
TABLE 2: Effect of farnesol and tyrosol on total protease production in Egyptian isolates of P. aeruginosa.

\begin{tabular}{|c|c|c|c|c|c|}
\hline \multirow[b]{2}{*}{ Isolate number } & \multirow{2}{*}{$\begin{array}{c}\text { Control } \\
\text { Total protease production } \\
\text { by the isolate }(\mathrm{U} / \mathrm{mL})\end{array}$} & \multicolumn{2}{|c|}{ Tyrosol effect } & \multicolumn{2}{|c|}{ Farnesol effect } \\
\hline & & $\begin{array}{c}\text { Before } \\
\text { normalization }\end{array}$ & $\begin{array}{c}\text { After } \\
\text { normalization }\end{array}$ & $\begin{array}{c}\text { Before } \\
\text { normalization }\end{array}$ & After normalization \\
\hline 1 & 107 & 75.6 & -29.3 & 89 & -16.8 \\
\hline 2 & 95 & 61 & -35.8 & 57.5 & 39.5 \\
\hline 3 & 100 & 88.5 & -11.5 & 80 & -20 \\
\hline 4 & 60.8 & 57.3 & -5.6 & 10.6 & 82.5 \\
\hline 5 & 198.8 & 154.6 & -22.2 & 102 & -48.7 \\
\hline 6 & 22 & 21 & -4.5 & 22 & -0.1 \\
\hline 7 & 76 & 60.8 & -20 & 64 & -15.8 \\
\hline 8 & 102.8 & 85.9 & -16.4 & 102 & -0.8 \\
\hline 9 & 128.9 & 118.2 & -8.3 & 96 & -25.5 \\
\hline 10 & 147.9 & 115.4 & -22 & 121.8 & -17.6 \\
\hline 11 & 65.5 & 54.2 & -17.3 & 65 & 0.8 \\
\hline 12 & 127 & 76.4 & -39.8 & 165.4 & -30.2 \\
\hline 13 & 33.5 & 46.4 & 38.5 & 32 & -4.5 \\
\hline 14 & 94.5 & 78.4 & -17 & 75.5 & 20.1 \\
\hline 15 & 160 & 91.3 & -42.9 & 143 & -10.6 \\
\hline 16 & 63.7 & 61.8 & -3 & 62 & -2.7 \\
\hline 17 & 90 & 70.7 & -21.4 & 101.7 & 13 \\
\hline 18 & 109 & 100.8 & -7.5 & 99 & -9.2 \\
\hline 19 & 130.7 & 123.8 & -5.3 & 82 & -37.3 \\
\hline 20 & 163 & 101.5 & -37.7 & 150 & -8 \\
\hline
\end{tabular}

The values in the control column represent the levels of total protease production by the isolates ( $\mathrm{SD}=44.3)$, as determined in [27], without addition of farnesol and tyrosol. The values in the columns of tyrosol and farnesol stand for either nonnormalized values ( $\mathrm{SD}=40$ and 40.5 for tyrosol and farnesol, resp.) or normalized values. The latter values indicate the percent of either decrease (negative values) or increase (positive values) in the production of total protease after normalization to untreated samples (control). All values represent the mean of three independent experiments.

permeabilizing action of farnesol for gentamicin in some strains of $S$. aureus reported by Jabra-Rizk et al. [26].

Hemolysin and protease are considered to be important virulence factors of $P$. aeruginosa [37-39] which contribute to the pathogenesis of Pseudomonas infection. Our results indicate without doubt that tyrosol and farnesol strongly inhibit the production of hemolysin in almost all of the Egyptian isolates under investigation. Out of the 20 Egyptian isolates of $P$. aeruginosa, 16 strains showed more than $30 \%$ inhibition of hemolysin production after incubation with tyrosol $(1.2 \mu \mathrm{M})$, while in case of farnesol, 14 strains showed the same effect. In comparison with hemolysin results, we observed that tyrosol inhibited to much lower extent the production of total proteases in the same investigated isolates, where 13 stains showed more than $10 \%$ inhibition in the production of such virulence factor, whereas no significant inhibitory effect could be observed after incubation of the isolates with farnesol $(10 \mu \mathrm{M})$. Previous reports demonstrated that farnesol inhibited the virulence factor lipase in S. aureus [40]. However, no studies have investigated the effect of tyrosol on any of the bacterial virulence factors.

In conclusion, despite their difference in chemical structure, our results indicate that the C. albicans QS compounds, tyrosol and farnesol, inhibit the production of hemolysin by $P$. aeruginosa. Additionally, the production of protease by
P. aeruginosa was repressed in the presence of tyrosol; however no significant decrease was observed in case of farnesol. Together with our novel observation of the antibacterial activity of tyrosol against $P$. aeruginosa, a suggestion for effective antimicrobial strategy may be broadly applicable to other bacterial species. Therefore, our research highlights the importance of studying effect of C. albicans QS compounds on $P$. aeruginosa as an example for bacterial pathogen that can coexist in mixed-species of microbial communities and might play an important role in vivo. Further studies are now in progress to identify the mechanism of inhibition of these virulence factors by tyrosol particularly the expression of hemolysin gene.

\section{Conflict of Interests}

The authors declare that there is no conflict of interests regarding the publication of this paper.

\section{References}

[1] M. B. Miller and B. L. Bassler, "Quorum sensing in bacteria," Annual Review of Microbiology, vol. 55, pp. 165-199, 2001.

[2] B. L. Bassler, "Small talk. Cell-to-cell communication in bacteria," Cell, vol. 109, no. 4, pp. 421-424, 2002. 
[3] J. M. Hornby, E. C. Jensen, A. D. Lisec et al., "Quorum sensing in the dimorphic fungus Candida albicans is mediated by farnesol," Applied and Environmental Microbiology, vol. 67, no. 7, pp. 2982-2992, 2001.

[4] H. Chen, M. Fujita, Q. Feng, J. Clardy, and G. R. Fink, “Tyrosol is a quorum-sensing molecule in Candida albicans," Proceedings of the National Academy of Sciences of the United States of America, vol. 101, no. 14, pp. 5048-5052, 2004.

[5] K. Weber, R. Sohr, B. Schulz, M. Fleischhacker, and M. Ruhnke, "Secretion of E, E-farnesol and biofilm formation in eight different Candida species," Antimicrob Agents Chemother, vol. 52, no. 5, pp. 1859-1861, 2008.

[6] N. Décanis, N. Tazi, A. Correia, M. Vilanova, and M. Rouabhia, "Farnesol, a fungal quorum-sensing molecule triggers Candida Albicans morphological changes by downregulating the expression of different secreted aspartyl proteinase genes," Open Microbiology Journal, vol. 5, pp. 119-126, 2011.

[7] M. A. S. Alem, M. D. Y. Oteef, T. H. Flowers, and L. J. Douglas, "Production of tyrosol by Candida albicans biofilms and its role in quorum sensing and biofilm development," Eukaryotic Cell, vol. 5, no. 10, pp. 1770-1779, 2006.

[8] D. K. Morales and D. A. Hogan, "Candida albicans interactions with bacteria in the context of human health and disease," PLoS Pathogens, vol. 6, no. 4, Article ID e1000886, 2010.

[9] E. Carlson, "Effect of strain of Staphylococcus aureus on synergism with Candida albicans resulting in mouse mortality and morbidity," Infection and Immunity, vol. 42, no. 1, pp. 285-292, 1983.

[10] M. E. Shirtliff, B. M. Peters, and M. A. Jabra-Rizk, "Crosskingdom interactions: Candida albicans and bacteria," FEMS Microbiology Letters, vol. 299, no. 1, pp. 1-8, 2009.

[11] L. J. Holcombe, G. McAlester, C. A. Munro et al., "Pseudomonas aeruginosa secreted factors impair biofilm development in Candida albicans," Microbiology, vol. 156, no. 5, pp. 1476-1485, 2010.

[12] L. J. Douglas, "Candida biofilms and their role in infection," Trends in Microbiology, vol. 11, no. 1, pp. 30-36, 2003.

[13] A. Neidig, A. T. Yeung, T. Rosay et al., “TypA is involved in virulence, antimicrobial resistance and biofilm formation in Pseudomonas aeruginosa," BMC Microbiology, vol. 13, no. 1, article 77, 2013.

[14] A. Bauernfeind, G. Hörl, R. Jungwirth et al., "Qualitative and quantitative microbiological analysis of sputa of 102 patients with cystic fibrosis," Infection, vol. 15, no. 4, pp. 270-277, 1987.

[15] C. Hermann, J. Hermann, U. Munzel, and R. Rüchel, "Bacterial flora accompanying Candida yeasts in clinical specimens," Mycoses, vol. 42, no. 11-12, pp. 619-627, 1999.

[16] M. A. El-Azizi, S. E. Starks, and N. Khardori, "Interactions of Candida albicans with other Candida spp. and bacteria in the biofilms," Journal of Applied Microbiology, vol. 96, no. 5, pp. 1067-1073, 2004.

[17] S. Nseir, E. Jozefowicz, B. Cavestri et al., "Impact of antifungal treatment on Candida-Pseudomonas interaction: a preliminary retrospective case-control study," Intensive Care Medicine, vol. 33, no. 1, pp. 137-142, 2007.

[18] J. Gibson, A. Sood, and D. A. Hogan, "Pseudomonas aeruginosa-Candida albicans interactions: localization and fungal toxicity of a phenazine derivative," Applied and Environmental Microbiology, vol. 75, no. 2, pp. 504-513, 2009.

[19] J. R. Kerr, "Suppression of fungal growth exhibited by Pseudomonas aeruginosa," Journal of Clinical Microbiology, vol. 32, no. 2, pp. 525-527, 1994.
[20] J. R. Kerr, G. W. Taylor, A. Rutman, N. Høiby, P. J. Cole, and R. Wilson, "Pseudomonas aeruginosa pyocyanin and 1hydroxyphenazine inhibit fungal growth," Journal of Clinical Pathology, vol. 52, no. 5, pp. 385-387, 1999.

[21] D. A. Hogan and R. Kolter, "Pseudomonas-Candida interactions: an ecological role for virulence factors," Science, vol. 296, no. 5576, pp. 2229-2232, 2002.

[22] D. A. Hogan, Å. Vik, and R. Kolter, "A Pseudomonas aeruginosa quorum-sensing molecule influences Candida albicans morphology," Molecular Microbiology, vol. 54, no. 5, pp. 12121223, 2004.

[23] G. E. Pierce, "Pseudomonas aeruginosa, Candida albicans, and device-related nosocomial infections: implications, trends, and potential approaches for control," Journal of Industrial Microbiology and Biotechnology, vol. 32, no. 7, pp. 309-318, 2005.

[24] C. Cugini, M. W. Calfee, J. M. Farrow III, D. K. Morales, E. C. Pesci, and D. A. Hogan, "Farnesol, a common sesquiterpene, inhibits PQS production in Pseudomonas aeruginosa," Molecular Microbiology, vol. 65, no. 4, pp. 896-906, 2007.

[25] L. S. Derengowski, C. De-Souza-Silva, S. V. Braz et al., "Antimicrobial effect of farnesol, a Candida albicans quorum sensing molecule, on Paracoccidioides brasiliensis growth and morphogenesis," Annals of Clinical Microbiology and Antimicrobials, vol. 8, article 13, 2009.

[26] M. A. Jabra-Rizk, T. F. Meiller, C. E. James, and M. E. Shirtliff, "Effect of farnesol on Staphylococcus aureus biofilm formation and antimicrobial susceptibility", Antimicrobial Agents and Chemotherapy, vol. 50, no. 4, pp. 1463-1469, 2006.

[27] S. H. M. Abdel-Rhman, A. M. El-Mahdy, and E. S. A. Abdelmegeed, "Optimization of protease production by Ps. Aeruginosa PAO1 and Physico-chemical characterization of the enzyme," Journal of American Science, vol. 10, no. 7, pp. 62-72, 2014.

[28] G. Rossignol, A. Merieau, J. Guerillon et al., "Involvement of a phospholipase $\mathrm{C}$ in the hemolytic activity of a clinical strain of Pseudomonas fluorescens," BMC Microbiology, vol. 8, article 189, 2008.

[29] D. K. Morales, N. J. Jacobs, S. Rajamani, M. Krishnamurthy, J. R. Cubillos-Ruiz, and D. A. Hogan, "Antifungal mechanisms by which a novel Pseudomonas aeruginosa phenazine toxin kills Candida albicans in biofilms," Molecular Microbiology, vol. 78, no. 6, pp. 1379-1392, 2010.

[30] D. K. Morales, N. Grahl, C. Okegbe, L. E. P. Dietrich, N. J. Jacobs, and D. A. Hogan, "Control of Candida albicans metabolism and biofilm formation by Pseudomonas aeruginosa phenazines," mBio, vol. 4, no. 1, Article ID e00526-12, 2013.

[31] C. Cugini, D. K. Morales, and D. A. Hogan, "Candida albicansproduced farnesol stimulates Pseudomonas quinolone signal production in LasR-defective Pseudomonas aeruginosa strains," Microbiology, vol. 156, no. 10, pp. 3096-3107, 2010.

[32] G. McAlester, F. O’Gara, and J. P. Morrissey, "Signal-mediated interactions between Pseudomonas aeruginosa and Candida albicans," Journal of Medical Microbiology, vol. 57, no. 5, pp. 563$569,2008$.

[33] M. L. Langford, A. L. Atkin, and K. W. Nickerson, "Cellular interactions of farnesol, a quorum-sensing molecule produced by Candida albicans," Future Microbiology, vol. 4, no. 10, pp. 1353-1362, 2009.

[34] H. Koo, P. L. Rosalen, J. A. Cury, Y. K. Park, and W. H. Bowen, "Effects of compounds found in propolis on Streptococcus mutans growth and on glucosyltransferase activity," 
Antimicrobial Agents and Chemotherapy, vol. 46, no. 5, pp. 13021309, 2002.

[35] F. I. A. Gomes, P. Teixeira, J. Azeredo, and R. Oliveira, "Effect of farnesol on planktonic and biofilm cells of Staphylococcus epidermidis," Current Microbiology, vol. 59, no. 2, pp. 118-122, 2009.

[36] H.-L. Alakomi, A. Paananen, M.-L. Suihko, I. M. Helander, and M. Saarela, "Weakening effect of cell permeabilizers on gram-negative bacteria causing biodeterioration," Applied and Environmental Microbiology, vol. 72, no. 7, pp. 4695-4703, 2006.

[37] D. E. Woods, M. S. Schaffer, H. R. Rabin, G. D. Campbell, and P. A. Sokol, "Phenotypic comparison of Pseudomonas aeruginosa strains isolated from a variety of clinical sites," Journal of Clinical Microbiology, vol. 24, no. 2, pp. 260-264, 1986.

[38] V. Majtán, A. Hostacká, A. Kosiarová, L. Majtánová, and S. Kohútová, "On the toxinogenicity of Pseudomonas aeruginosa strains," Journal of Hygiene, Epidemiology, Microbiology, and Immunology, vol. 35, no. 2, pp. 217-224, 1991.

[39] J. L. Kadurugamuwa and T. J. Beveridge, "Virulence factors are released from Pseudomonas aeruginosa in association with membrane vesicles during normal growth and exposure to gentamicin: a novel mechanism of enzyme secretion," Journal of Bacteriology, vol. 177, no. 14, pp. 3998-4008, 1995.

[40] M. Kuroda, S. Nagasaki, R. Ito, and T. Ohta, "Sesquiterpene farnesol as a competitive inhibitor of lipase activity of Staphylococcus aureus," FEMS Microbiology Letters, vol. 273, no. 1, pp. 28-34, 2007. 

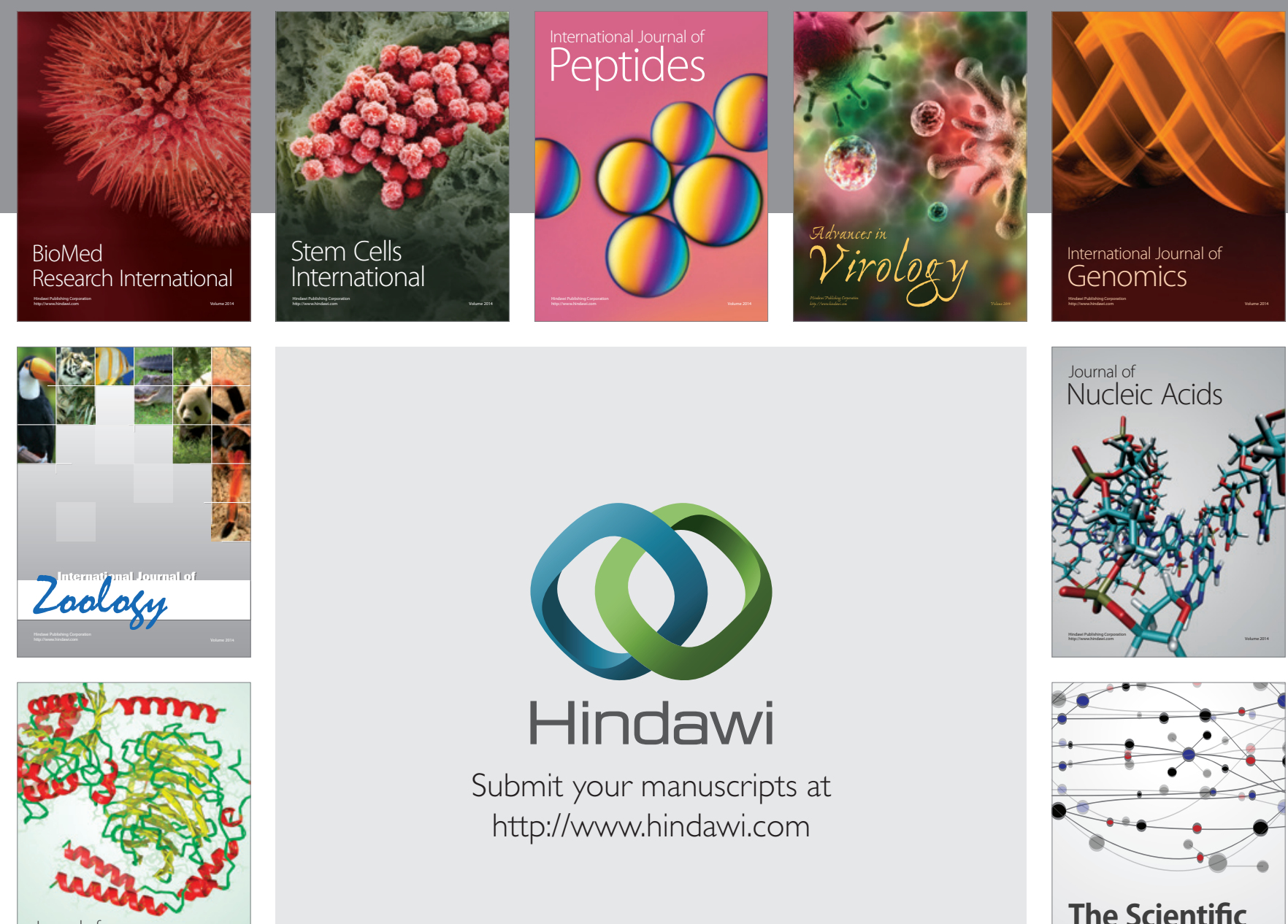

Submit your manuscripts at

http://www.hindawi.com

Journal of
Signal Transduction
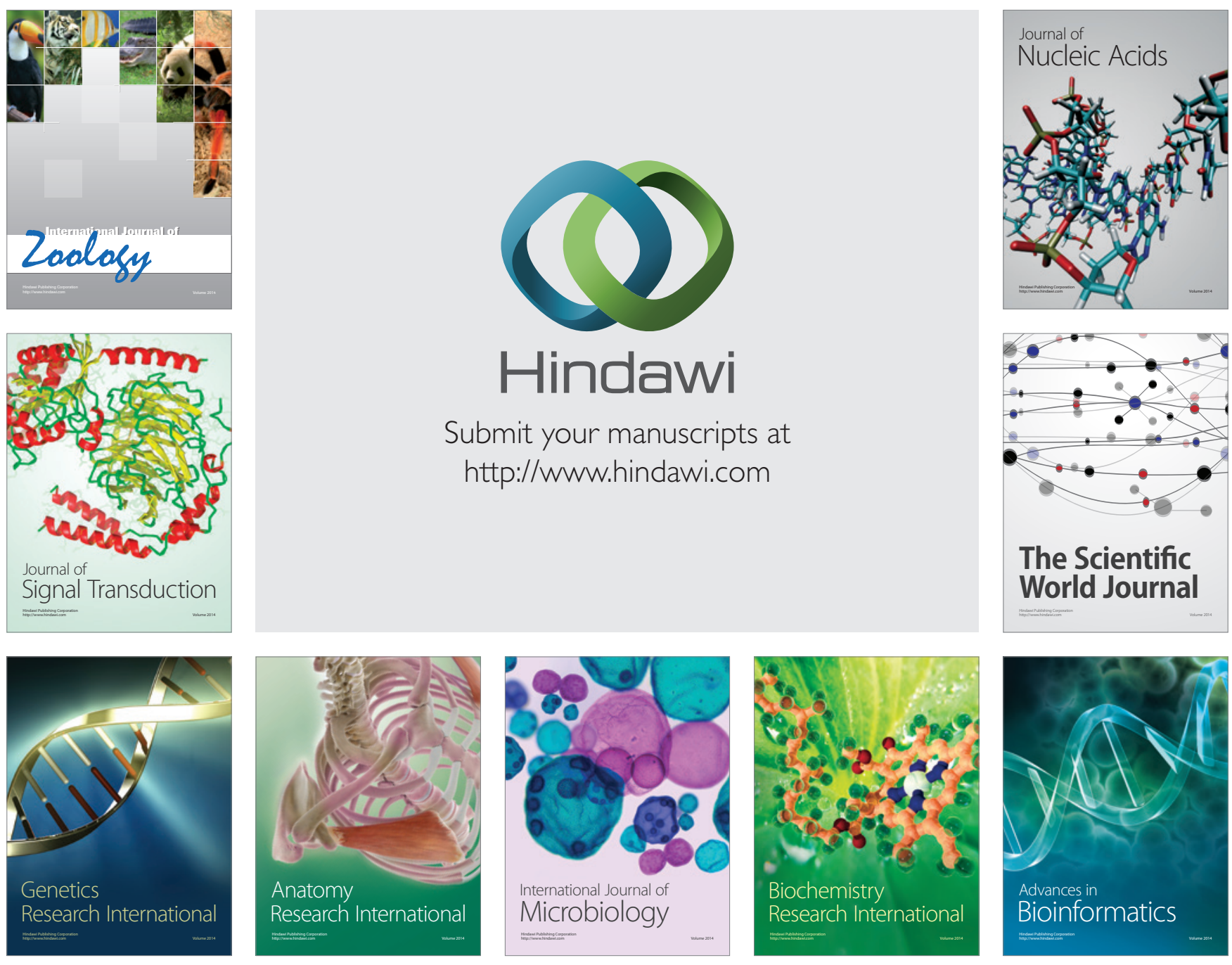

The Scientific World Journal
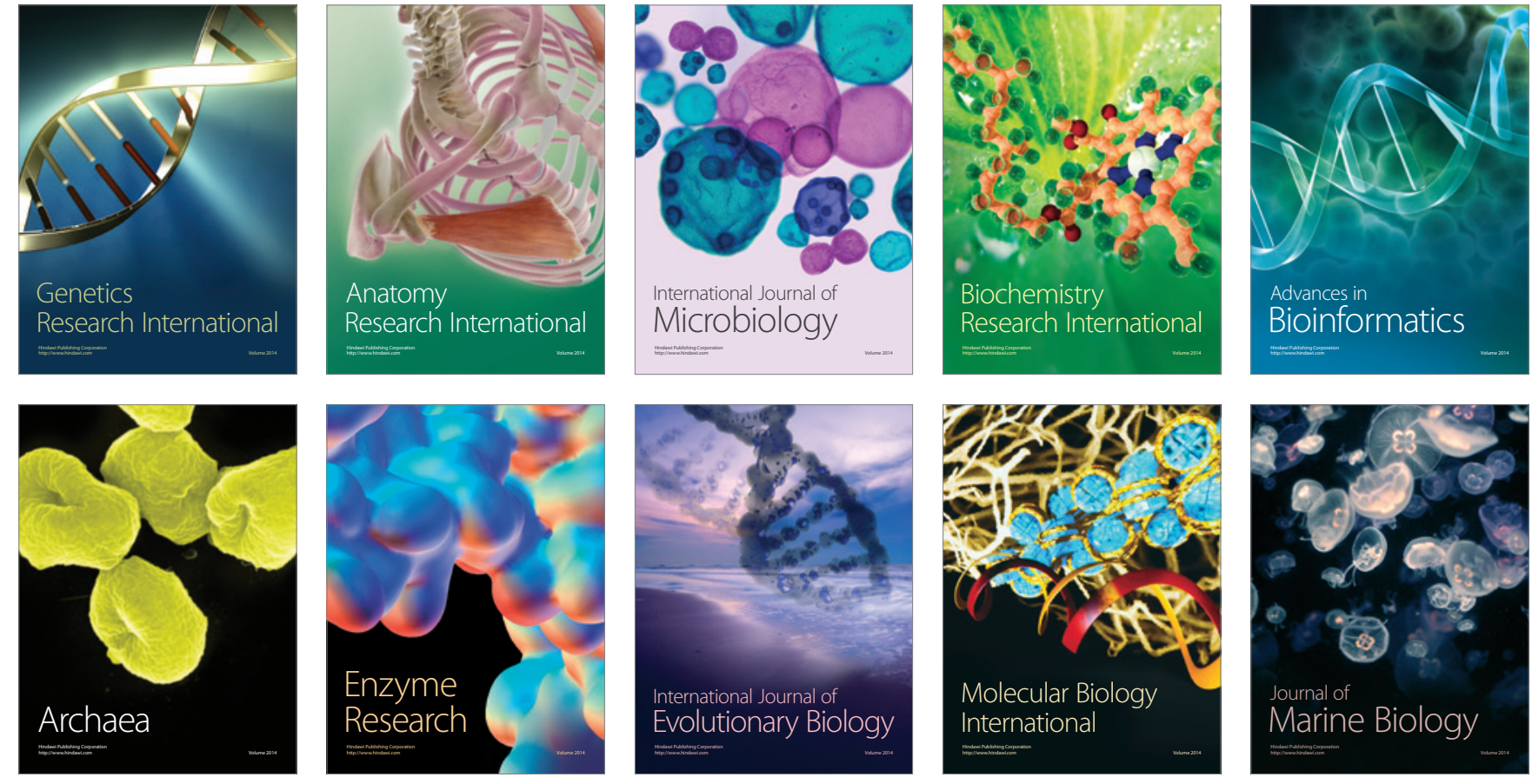